\section{Verhaltenstherapie}

\title{
Isaac Marks: «Wir brauchen eine gemeinsame Sprache für Psychotherapieverfahren»
}

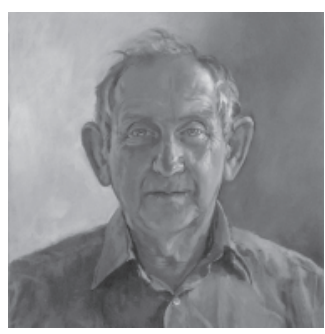

Dr. med. Isaac Marks ist Professor Emeritus für experimentelle Psychopathologie am Maudsley Hospital / Institute of Psychiatry in London. Er ist der "Vater der Expositionstherapie» für Angst- und Zwangsstörungen. Als unermüdlicher Therapieforscher untersuchte er vor allem folgende Fragestellung: Welches sind die unverzichtbaren, aber auch hinreichenden Maßnahmen, um möglichst in Kurzzeittherapien Veränderungen beim Patienten einzuleiten, die dessen Selbsthilfefertigkeiten soweit verbessern, dass weiterer Therapiefortschritt mit minimaler therapeutischer Unterstützung, z.B. via Telefon oder Computer möglich wird? Marks ist der Begründer des Ausbildungsprogramms für "Behavioural Nurse-Therapists", das in Großbritannien vor über drei Jahrzehnten begonnen wurde und inzwischen Hunderte von NurseTherapists hervorgebracht hat, die sowohl in stationären Einrichtungen als auch ambulant, an praktische Ärzte angeschlossen, als Verhaltenstherapeuten arbeiten - und das mit gleich guten Ergebnissen wie psychologische und psychiatrische Verhaltenstherapeuten. Isaac Marks hat 12 Bücher und 440 wissenschaftliche Artikel veröffentlicht. Er war Berater der WHO und des National Institute for Mental Health (USA), Fellow am Center for Advanced Study in the Behavioral Sciences an der Stanford-Universität in Kalifornien und hat mehrere Preise für die Entwicklung von computerunterstützten Selbsthilfeverfahren für Agoraphobie, Panikstörung und Zwangsstörungen erhalten. Das Interview führte Iver Hand, selbst Schüler von Isaac Marks (1971-1973), via E-Mail.

Betrachtet man die historische Entwicklung der Verhaltenstherapie, so überrascht es, welche entscheidende Rolle südafrikanische Psychologen (Rachmann, Lazarus, Wilson) und Ärzte (Wolpe und du) bereits in den frühen Jahren der Verhaltenstherapie gehabt haben. Hast du eine Erklärung dafür?

Isaac Marks: Das mag reiner Zufall sein. Möglicherweise haben uns einige Aspekte der südafrikanischen Weltanschauung beeinflusst. Allerdings haben die anderen ihre Ausbildung an der Wittwatersrand Universität (WITS) in Johannesburg gemacht, während ich mein Medizinstudium 1500 km entfernt an der Universität von Kapstadt absolviert habe und nicht wusste, was an der WITS gelehrt wurde. Ich bekam an meiner Universität vor allem die medizinische Tradition des British Empire mit. Diese Tradition war auch am Maudsley Hospital in London sehr ausgeprägt, wo ich 1963 meine psychiatrische Weiterbildung abschloss. Die dortige, ausgeprägt experimentelle Orientierung zog mich sehr an. Verhaltenstherapie schien dort gut hineinzupassen, obwohl dann nach und nach deutlich wurde, dass die Verhaltenstherapie und später die kognitive Therapie wie fast alle Therapien von Ideologisierung geprägt waren. Bis zu meiner gemeinsamen Verhaltenstherapieforschung mit Michael Gelder am Maudsley Hospital hatte ich keinen Kontakt zu Rachmann, Lazarus, Wilson oder Wolpe.

Jahrzehntelang hast du immer wieder Beiträge zur Frage der relativen Bedeutung behavioraler versus kognitiver Interventionen für die Veränderung von Krankheitsverhalten geleistet. Findest $d u$, dass heute immer noch kontroverse Positionen im Vordergrund stehen oder haben wir eine Synthese oder vielleicht sogar einen neuen Denkansatz erreicht, z.B. durch die neuerlich entdeckte Bedeutung der Emotion, wie man Menschen am besten helfen kann, sich zu verändern?

Isaac Marks: Es gibt immer noch kontroverse Debatten, aber sie haben sich verändert. Jahrzehntelang beinhaltete kognitive Therapie auch die Anwendung von Verhaltenstherapie. Lange blieb offen, in welchem Ausmaß kognitive Interventionen zusätzlichen oder eigenständigen Erfolg brachten. Es ist kogniti-

\begin{tabular}{|c|c|}
\hline KARGER & (C) 2005 S. Karger GmbH, Freiburg \\
\hline $\begin{array}{l}\text { Fax +4976145207 } 14 \\
\text { E-mail Information@Karger.de } \\
\text { www.karger.com }\end{array}$ & $\begin{array}{l}\text { Accessible online at: } \\
\text { www.karger.com/ver }\end{array}$ \\
\hline
\end{tabular}

Herrn Prof. Dr. Iver Hand

Universitätskrankenhaus Eppendorf, Psychiatrie/Verhaltenstherapie

Martinistraße 52, 20251 Hamburg, Deutschland

Tel. +49 40 42803-2233, Fax -5025

E-mail hand@uke.uni-hamburg.de 
ven Therapeuten nicht gelungen, Studien zu entwickeln und durchzuführen, die diese Frage hätten klären können. In letzter Zeit kamen allerdings einige Studien zu dem Ergebnis, dass kognitive Therapie zwar keinen zusätzlichen oder gar überlegenen Effekt im Vergleich zur Verhaltenstherapie hat, aber durchaus auch ohne verhaltenstherapeutischen Inhalt wirksam sein kann. Allerdings ist bis heute nicht geklärt, ob kognitive Therapie dadurch wirkt, dass sie Kognitionen umstrukturiert (es gibt eher Hinweise, dass dies nicht der Fall ist), oder ob andere Wirkmechanismen vorliegen. Analog könnte es durchaus sein, dass Exposition als verhaltenstherapeutisches Verfahren zwar zur Habituation von Angst führt, dass uns aber auch dabei die eigentlichen Wirkmechanismen noch unklar sind. Eine langsam wachsende Anzahl von Verfahren, die wir üblicherweise weder der Verhaltens- noch der kognitiven Therapie zurechnen, scheint ebenfalls zu einer Verbesserung von Angst und Depression zu führen, zum Beispiel Atemtraining, Aufmerksamkeitstraining, Meditation (die die beiden vorherigen Verfahren beinhaltet) oder induzierte Anspannung. Wissenschaftliche Erklärungen für Veränderungsmechanismen in verschiedenen, wirksamen Therapien sind nur dann überzeugend, wenn sie auf alle Behandlungen anwendbar sind. Eine solche Synthese in Richtung auf ein neues Paradigma der Psychotherapie ist aber in näherer Zukunft kaum zu erwarten. Es ist zu hoffen, dass es nicht die 350 Jahre dauert, die verstrichen sind, bevor aus den Experimenten der alten Griechen mit statischer Elektrizität die elektrischen und elektronischen Geräte möglich wurden, die unsere moderne Welt prägen.

In den frühen 70er Jahren war Exposition in vivo in deiner Abteilung bereits die Standardmethode zur Behandlung der Symptome von Angsterkrankungen (sogar in Gruppen) und Zwangsstörungen. Im Jahr 2003 hielt die «Oxford-Gruppe» auf der Jahrestagung der Europäischen Gesellschaft für Verhaltensund kognitive Therapie in Prag ein Symposium über «Die Bedeutung der Exposition in der kognitiven Therapie von Angststörungen». Ein Highlight dieses Symposiums war ein Video eines BBC-Fernsehfilms, in dem Paul Salkovskis, jetzt Professor am Maudsley Hospital, eine einzelne Patientin auf einer Expositionssitzung in einem Kaufhaus begleitete. Dies wurde als neuester Fortschritt in der kognitiven Therapie dargestellt. Wie empfindest du das? Was läuft da in unserem Fach ab?

Isaac Marks: Das ist nicht das erste Mal, dass Therapeuten gut untersuchte und etablierte Behandlungsverfahren lange Zeit ignorieren, um dann irgendwann das Rad neu zu erfinden. Invivo-Exposition war in der Tat seit Anfang der 1950er Jahre eine häufig benutzte behaviorale Methode unter Namen wie Gestuftes Üben, Habituation, Sukzessive Annäherung, Löschung, Konfrontation, Flooding, In-vivo- oder Kontaktdesensibilisierung, Modeling, Guided Mastery etc. In meiner Abteilung ist Exposition in vivo seit 1970 die Standardtherapie für Angststörungen, auch unter diesem Namen. In meinem Arti- kel «The Reduction of Fear: Towards a Unifying Theory» habe ich 1973 dargestellt, dass alle diese unterschiedlich bezeichneten Methoden letztlich Exposition beinhalten. Ebenfalls schon seit den 50er Jahren wurden in Tierstudien Expositionsverfahren unter verschiedenen Namen eingesetzt, um Vermeidung und Furchtreaktionen zu löschen. Seit den 80er Jahren haben kognitive Therapeuten Expositionen als «Verhaltensexperimente» oder «Infragestellungen irrationaler Überzeugungen» bezeichnet. Dieses Beispiel zeigt sehr deutlich, dass wir unbedingt die begriffliche Konfusion in unserem Fach reduzieren müssen und eine gemeinsame Sprache für Psychotherapieverfahren benötigen. Aus diesem Grund haben die EABCT (European Association of Behavioural and Cognitive Therapy) und die AABT (Association for the Advancement of Behavior Therapy) eine gemeinsame Arbeitsgruppe eingesetzt. Ich bin zu ihrem Vorsitzenden gewählt worden. Bereits in der Anfangsphase wurden eine ganze Reihe von Erkenntnissen gewonnen, aber es wird noch Jahre dauern, bis aus dem Projekt einer «gemeinsame Sprache» ein Wörterbuch entstanden sein wird, das die meisten Psychotherapeuten als Standard akzeptieren, um zu beschreiben, was sie tun - nicht, warum sie es tun. Um dieses Ziel zu erreichen, werden die Egos, die gegenwärtig in diesem Prozess im Dunkeln immer noch aneinander stoßen, hoffentlich nach und nach akzeptieren, dass Psychotherapie nur dann eine Wissenschaft mit replizierbaren Resultaten werden kann, wenn eine breit akzeptierte Nomenklatur (möglichst mit Angabe, wie und wo der jeweilige Begriff erstmals benutzt wurde) besteht.

Du bist am Maudsley im Jahr 2000 emeritiert worden, aber so aktiv wie eh und je. Was werden deine Beiträge für unser Fach in den kommenden Jahren sein?

Isaac Marks: Mit Studenten forsche ich weiter an Fragestellungen der computerunterstützten kognitiven Verhaltenstherapie (Computer-Assisted Cognitive Behaviour Therapy; CCBT). Außerdem schreibe ich an einem Maudsley-Monograph (mein erstes Buch, 1965, war auch ein Maudsley-Monograph), einem Review der internationalen Literatur zu CCBTSelbsthilfe. Das ist sehr spannend, denn neue, wichtige Artikel erscheinen in einem solchen Tempo, dass sie kaum noch adäquat rezipiert und in die vorhandene Literatur integriert werden können. Eine zweite, noch größere und länger dauernde Aufgabe ist die schon erwähnte EABCT-AABT-Arbeitsgruppe zur Entwicklung eines Wörterbuchs für eine gemeinsame Sprache der Psychotherapie. Wenn deine Leser dazu unter ihrem Namen jeweils ein oder zwei Beiträge erstellen möchten, können sie gerne die Richtlinien für die Manuskripterstellung zugeschickt bekommen.

Du hast nun vier Jahrzehnte äußerst aktiv die Entwicklung der Verhaltenstherapie mitgestaltet und analysierst ihren aktuellen Stand - wo siehst du unser Fach in den nächsten fünf bis zehn Jahren? 
Isaac Marks: Die Zukunft aus der Kristallkugel vorhersagen zu wollen, kann sehr in die Irre führen. 1970 sagte ich in einem Artikel, «The Future of the Psychotherapy», voraus, dass sich die verschiedenen Psychotherapieschulen zusammentun würden, aber das lässt sich immer noch nicht erkennen. Möglicherweise setzt sich aber die Erkenntnis durch, dass es mehrere gute Wege gibt (neben den Standardmethoden der Verhaltens- und kognitiven Therapie), Angst und Depression zu reduzieren. Aus einer Gesamtschau könnten dann Konzepte entstehen, wie die Krankheitssymptomatik am besten reduziert werden kann. Die Zusammenarbeit von Forschern aus der kognitiven Verhaltenstherapie und der Bildgebung könnte das Verstehen von Veränderungsmechanismen beschleunigen. Computerunterstützte kognitive Verhaltenstherapie könnte verstärkt in die tägliche Routineversorgung eingebaut werden und so erheblich mehr Betroffenen helfen. Daraus könnte sich die erstmalige Chance eröffnen, die Prävalenz von Angstund depressiven Störungen in der Bevölkerung in einem realistischen Kostenrahmen zu reduzieren. Eine weitere positive Entwicklung könnte aus dem gegenwärtigen Lippenbekenntnis zur evidenzbasierten Praxis resultieren. Vielleicht werden irgendwann auch die Gesundheitspolitiker und Versicherungen die professionellen Therapeuten dazu bringen, routinemäßig Daten über die Fortschritte ihrer Patienten und die dabei entstehenden Kosten zu erstellen. Computerisierte Bilanzsysteme könnten diesen Prozess unterstützen bis hin zu einem internationalen «Clearinghouse of Clinical Outcome», das dann den Klinikern einen internationalen Vergleich ihrer Ergebnisse ermöglichen würde - vorausgesetzt, dass Gleiches mit Gleichem verglichen wird. Die Therapeuten werden sich aber vielleicht einer regelmäßigen Überprüfung entziehen wollen. Wir würden ja auch nicht erwarten, dass Truthähne sich für die Beibehaltung von Weihnachten aussprechen würden. Würde sie aber als Bedingung für die Bezahlung von Therapie eingeführt, dann hätte dieses sicher positive Auswirkungen auf das Denken und Handeln von Therapeuten. Dies entspräche auch anderen Entwicklungen im Gesundheitswesen, wie zum Beispiel in England, wo die Behandlungen von Zahnärzten oder Todesfälle nach chirurgischen Eingriffen extern überprüft werden.

Reden wir jetzt nicht über Veränderungen, die vielleicht in 50 oder 500 Jahren statt in den nächsten 5 Jahren kommen können?

Isaac Marks: Schon seit 1990 haben wir in meiner Abteilung am Maudsley unsere Behandlungsergebnisse routinemäßig überprüft und bei Hunderten von Patienten dabei unser Computerprogramm CORM (Clinical Outcome Monitoring and Resource Measure) zur Erfassung von Therapieergebnis und Therapieaufwand eingesetzt. Auch in den 20 Jahren davor haben wir, im weniger effizienten Papier- und Bleistiftverfahren, regelmäßig Therapieziele, Hauptproblembereiche und Therapieergebnis einschließlich der privaten und beruflichen Auswirkungen erfasst.
Wir haben aber doch selbst innerhalb des Feldes der Psychotherapieforschung noch kaum Studien, die die Cochrane-Kriterien erfüllen. Darüber hinaus wissen wir aus klinischer Erfahrung, dass der Therapieaufwand, z.B. bei Agoraphobie mit Panikstörung, extrem variieren kann: von bloßer Psychoedukation durch Literatur oder Internet über einige wenige Therapeutenkontakte bis hin zu 50 oder mehr therapeutengeleiteten Sitzungen einschließlich Exposition in vivo - und selbst dann mag der Erfolg ausbleiben. Was sind deiner Meinung nach die Variablen, die für diese extremen Unterschiede im Therapiebedarf und im Therapieergebnis verantwortlich sind?

Isaac Marks: Die große Mehrzahl der Patienten mit Agoraphobie und Panik kann mit 1,5 Stunden Therapeutenkontakt in 10 Wochen erfolgreich behandelt werden, wenn routinemäBige Anleitung zur Selbstexposition mit dem computerunterstützten Selbsthilfesystem «FearFighter» eingesetzt wird. Ohne diese Unterstützung lag die durchschnittliche Stundenzahl des direkten Therapeut-Patienten-Kontakts zur Unterstützung von Selbsthilfeexposition bei Agoraphobie und Panik in meiner Abteilung bei 8 Stunden. Das Gesetz der abnehmenden Effizienz (Law of Diminishing Returns) gilt mit Sicherheit, wenn bei diesen Patienten mehr als 20 Sitzungen aufgewendet werden; es sei denn, es gibt klare Hinweise darauf, dass die Fortsetzung der Therapie zu eindeutigen zusätzlichen Erfolgen führt. 50 Sitzungen sind normalerweise Zeitverschwendung. In dieser Zeit könnte der Therapeut 6 bis 25 andere Patienten mit mehr Erfolg behandeln.

Um das Problem noch einmal zu verkomplizieren: Metaanalysen von Therapiestudien kommen nicht selten zu unterschiedlichen Schlussfolgerungen, weil sich die analysierten Studien bezüglich Messinstrumenten, Designs, Therapieverfahren (selbst bei identischen Bezeichnungen) und Ergebniskriterien unterscheiden. Welchen Typ von Evidenz soll der Psychotherapiepraktiker also in Zukunft liefern, damit er bezahlt wird?

Isaac Marks: In Neuseeland wurde ein Facharzt für psychosomatische Medizin von der Versicherungsgesellschaft zusätzlich bezahlt, damit er für bestimmte Patienten Daten auf der Basis von CORM vorlegte. Es gibt keinen Grund, warum ein solches Prinzip der Bezahlung nicht im breiteren Umfang genutzt werden sollte.

\section{Herzlichen Dank für das Interview!}

Interview 